\title{
Peptide Interfacial Biomaterials Improve Endothelial Cell Adhesion and Spreading on Synthetic Polyglycolic Acid Materials
}

\author{
Xin Huang, ${ }^{1}$ Stefan Zauscher, ${ }^{2}$ Bruce Klitzman, ${ }^{1,3}$ George A. Truskey, ${ }^{1}$ William M. Reichert, ${ }^{1}$ \\ Daniel J. Kenan, ${ }^{4}$ and Mark W. GrinstafF ${ }^{5}$ \\ ${ }^{1}$ Department of Biomedical Engineering, Duke University, Durham, NC 27708, USA; ${ }^{2}$ Department of Mechanical Engineering \\ and Material Science, Duke University, Durham, NC 27708, USA; ${ }^{3}$ Kenan Plastic Surgery Research Labs, Duke University \\ Medical Center, Durham, NC 27710, USA; ${ }^{4}$ Department of Pathology, Duke University Medical Center, Durham, NC 27710 , \\ USA; and ${ }^{5}$ Departments of Biomedical Engineering and Chemistry, Boston University, Boston, MA 02215, USA
}

(Received 26 January 2010; accepted 23 February 2010; published online 19 March 2010)

Associate Editor Michael S. Detamore oversaw the review of this article.

\begin{abstract}
Resorbable scaffolds such as polyglycolic acid (PGA) are employed in a number of clinical and tissue engineering applications owing to their desirable property of allowing remodeling to form native tissue over time. However, native PGA does not promote endothelial cell adhesion. Here we describe a novel treatment with hetero-bifunctional peptide linkers, termed "interfacial biomaterials" (IFBMs), which are used to alter the surface of PGA to provide appropriate biological cues. IFBMs couple an affinity peptide for the material with a biologically active peptide that promotes desired cellular responses. One such PGA affinity peptide was coupled to the integrin binding domain, Arg-Gly-Asp (RGD), to build a chemically synthesized bimodular 27 amino acid peptide that mediated interactions between PGA and integrin receptors on endothelial cells. Quartz crystal microbalance with dissipation monitoring (QCMD) was used to determine the association constant $\left(K_{\mathrm{A}}\right.$ $\left.1 \times 10^{7} \mathrm{M}^{-1}\right)$ and surface thickness $(\sim 3.5 \mathrm{~nm})$. Cell binding studies indicated that IFBM efficiently mediated adhesion, spreading, and cytoskeletal organization of endothelial cells on PGA in an integrin-dependent manner. We show that the IFBM peptide promotes a $200 \%$ increase in endothelial cell binding to PGA as well as $70-120 \%$ increase in cell spreading from 30 to 60 minutes after plating.
\end{abstract}

Keywords-PGA, Surface modification, IFBM, Peptides, RGD, Biomaterials, Scaffolds.

\section{INTRODUCTION}

The surface properties of materials strongly influence the performance of implanted biomedical devices. $^{2,12,43,55}$ Existing FDA approved materials used in implantable devices are often limited in their

Address correspondence to Mark W. Grinstaff, Departments of Biomedical Engineering and Chemistry, Boston University, Boston, MA 02215, USA. Electronic mail: mgrin@bu.edu adhesional and bio-functional properties. For example, synthetic polymers currently used as artificial coronary artery bypass grafts have significantly lower patency rates when compared to those with autologous vein or artery grafts. ${ }^{18,33}$ This is possibly due to compliance mismatch and thrombogenicity, which are linked to incomplete re-endothelialization or dysfunction of the endothelial cells that do grow over the grafts. Niklason et al. employed polyglycolic acid (PGA), a biodegradable polymer, as scaffolding material and successfully engineered small diameter vascular grafts ${ }^{37}$; however, reendothelialization of these engineered vascular grafts with human endothelial cells still remains a major challenge. It is likely that surface modification of the inner PGA lining of a tissue engineered artery with biologically active coatings can restore endothelial cell function and thus improve device performance.

Surface modifications, including both chemical and topographical modifications, have been shown to influence protein adsorption, cell interaction, and host responses. ${ }^{42}$ Most synthetic biomaterials lack specific cellular and molecular interacting sites on their surfaces; instead, they non-specifically adsorb proteins from their surrounding environment. Vroman describes a transient and hierarchical adsorption of plasma proteins, including albumin and fibrinogen, when foreign materials are exposed to blood. ${ }^{52}$ Previous in vivo studies indicate that the non-specific protein adsorption onto the implanted biomaterials is possibly one of the key factors that trigger foreign body reactions. For instance, polymeric, ceramic, and metallic surfaces, regardless of significant surface differences such as hydrophilicity and softness, all trigger similar host responses. In contrast, protein-resistant surfaces, 
coated with nonfouling polymers such as polyethylene glycol (PEG), usually reduce inflammatory responses and elongate the life span of implanted devices. These findings suggest that an engineered surface modification system that selectively interacts with molecules, proteins, or cells has the potential to minimize unfavorable responses and to promote favorable responses. Biologically inspired materials that mimic the extracellular matrix (ECM) have drawn tremendous attention as model systems for the study of surface modification due to their ability to provide a plethora of biological cues that orchestrate complex biological responses. ${ }^{5,10,48}$

Polyglycolic acid and copolymers of glycolic and lactic acid are biodegradable and biocompatible polymers approved by the FDA for internal use. The degradation mechanism of PGA and its copolymers is well known. ${ }^{44}$ Due to its ester backbone linkage, in vivo degradation of PGA is influenced both by random hydrolysis and by enzymes including tissue esterases. PGA's degradation product, glycolic acid, is non-toxic and is metabolized through the tricarboxylic acid cycle. Because of its fast degradation rate and bioresorbability, PGA is widely applied in biomedical research and clinical use, such as in surgical sutures, drug delivery devices, and tissue engineering research. ${ }^{26}$

Untreated PGA surfaces perform poorly in promoting cell adhesion and proliferation, thus inspiring a number of strategies for PGA surface modification. A bio-adhesive glass, Bioglass ${ }^{\circledR}$, composed of $\mathrm{SiO}_{2}$, $\mathrm{Na}_{2} \mathrm{O}, \mathrm{CaO}$ and $\mathrm{P}_{2} \mathrm{O}_{5}$, has been used to modify PGA for bone engineering to increase microvascularization. ${ }^{9}$ Sodium hydroxide is also used to pre-treat PGA to enhance cell adhesion by breaking ester bonds and introducing carboxylic acid groups. ${ }^{14}$ Some RGD containing peptides, derived from human collagen type I, have been chemically grafted onto PGA surfaces and have demonstrated effectiveness for strengthening cell adhesion. ${ }^{13}$ However, these approaches either change the mechanical properties of the polymer, are non-trivial, or are costly, and the resulting modified surfaces lack the complexity of biological cues exhibited by native ECM.

Motivated by the unmet clinical need to promote efficient, robust, and thromboresistant re-endothelialization onto a number of intravascular devices, our group is designing and developing peptide-based coatings for PGA that mimic essential biological cues provided by ECM. We screened peptide phage display libraries, ${ }^{49}$ sampling more than 4 billion different peptides, to develop a subset of peptides with high affinity for PGA. Next, we used solid phase chemical synthesis to build a synthetic bimodular peptide incorporating a PGA-binding motif coupled to the tripeptide RGD that is known to bind certain integrin (a)
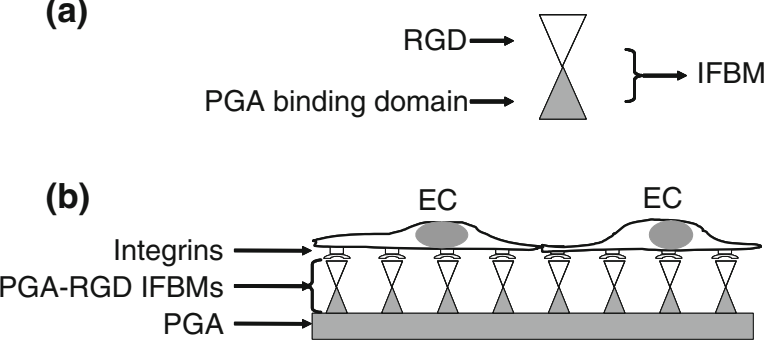

FIGURE 1. Interfacial biomaterials (IFBMs) as bio-adhesive material coatings. (a) Domain organization of an IFBM. The PGA binding domain consists of a peptide sequence derived by phage display panning of PGA material. The cell binding domain consists of an Arg-Gly-Asp (RGD) tripeptide sequence widely known to bind certain integrin receptors. These two domains are chemically synthesized as a single co-linear peptide. (b) PGA materials are coated with the PGA-RGD IFBM, where it can mediate matrix-like interactions with endothelial cells via integrin receptors.

receptors. RGD is a well characterized moiety found in matrix proteins such as fibronectin, laminin, and vitronectin where it influences cell adhesion, mobility, proliferation, and survival. ${ }^{13}$ In this paper, we present our work on engineering and characterizing a PGA-binding interfacial biomaterial (IFBM) peptide (Fig. 1) to promote EC adhesion and spreading.

\section{EXPERIMENTAL SECTION}

\section{PGA Panning}

PGA mesh (Concordia Fibers, LLC, formally Albany International) was cut into $2.5 \times 2.5 \mathrm{~cm}$ squares. Prior to the first round of panning, PGA square meshes were pre-incubated with $5 \%$ dry milk in phosphate buffered saline (M-PBS) to block nonspecific binding sites. The M-PBS block was alternated with 3\% bovine serum albumin (BSA, Sigma) block in PBS (BSA-PBS) in alternate rounds to discourage the recovery of block-binding phage. In the first round of panning, $1 \times 10^{11} \mathrm{pfu}$ of library was pre-cleared in $1 \mathrm{~mL}$ blocking solution in an empty well of a 6-well plate for $1 \mathrm{~h}$ to remove polystyrene binding phage. After pre-clearing, soluble phage in block solution were transferred to an adjacent well containing a PGA square for incubation. After 30-min incubation, PGA square mesh was removed to a clean tube and washed exhaustively with $0.5 \%$ Tween 20 (Sigma) in PBS (PBS-T) to remove non-binding phage. Mesh squares were then transferred to a $50 \mathrm{~mL}$ conical tube containing $5 \mathrm{~mL}$ of exponentially growing Escherichia coli TG-1 cells $\left(\mathrm{OD}_{600} 0.5-0.6\right)$ for recovery of phage by direct infection of E. coli. After $30 \mathrm{~min}$ of incubation without shaking, the tube was incubated with shaking for $1 \mathrm{~h}$, then mesh squares were removed from the tube 
and the infected bacteria incubated an additional $5 \mathrm{~h}$. Phage supernatants were recovered from bacterial cultures by centrifugation. Phage amplified for a given round of selection were used as input for the subsequent round in otherwise identical fashion.

\section{On-Phage ELISA}

On-phage Enzyme-Linked ImmunoSorbent Assays (ELISAs) were performed in 96-well pro-bind polystyrene plates (BD Falcon, Franklin Lakes, NJ). PGA mesh squares were cut and weighed in order to normalize ELISA results by the weight of PGA samples. PGA squares were treated with blocking buffer prior to binding assay. Both M-PBS and BSA-PBS were independently assayed in the absence of PGA squares as negative controls to rule out phage binding to plastic or to block. Approximately $1 \times 10^{10} \mathrm{pfu}$ of individual phage clones were added in a volume of $100 \mathrm{~mL}$ PBS-T into separate wells containing PGA plus block or block alone. Phage were allowed to bind for one hour, after which unbound phage were removed by exhaustive washing with PBS-T. Bound phage were detected by ELISA with anti-M13 antibody with HRP conjugate added at a dilution of 1:5000. PGA ELISA values were often of sufficiently high magnitude that dilutions were necessary to read within the linear range of the plate reader. ELISA values reported herein were corrected for dilution.

\section{DNA Sequencing}

Individual phage clones validated by multiple ELISA were chosen for further evaluation. Clones were grown in $5 \mathrm{~mL}$ cultures in $2 \mathrm{XYT}$ medium. Phage DNA was purified using the QIAprep spin M13 kit (QIAgen Inc). Purified DNA was quantified by $\mathrm{OD}_{260}$, treated with PIII reverse primer $^{20}$ and submitted for sequencing at the Duke Comprehensive Cancer Center DNA Sequencing Facility. Phage displayed peptide sequences were inferred from the resulting DNA sequences.

\section{Cell Culture}

Human umbilical vein endothelial cells (HUVECs; Cambrex) were grown to $80 \%$ confluence in $\mathrm{T}-25$ or $\mathrm{T}-75$ polystyrene flasks (BD) with endothelial basal media (EBM from Cambrex) supplemented with EGM (Cambrex; final FBS concentration 2\%). Cells were cultured with $5 \% \mathrm{CO}_{2} / 95 \%$ air at $37{ }^{\circ} \mathrm{C}$. HUVECs were used at passage 3 or 4 for all experiments. After rinsing HUVECs with Dulbecco's phosphate buffered saline (Gibco), 0.25\% trypsin was applied for $5 \mathrm{~min}$ at $37^{\circ} \mathrm{C}$. After cells were released, the trypsin was neutralized by adding an equal volume of EBM with
EGM ( $2 \%$ FBS). Cell suspension was immediately centrifuged at $1000 \mathrm{rpm}$ for $15 \mathrm{~min}$ and re-suspended in PBS for specific experiments.

\section{Cell Adhesion Assay on PGA Mesh}

PGA squares were sterilized by $70 \%$ ethanol and dried in a biological hood for $1 \mathrm{~h}$. Squares were then treated with $2 \mathrm{~mL}$ of $0.5 \mathrm{mg} / \mathrm{mL}$ of either PGARGD-(7-1) peptide as IFBM or PGA-RGE-(7-2) peptide as negative control in PBS for $1 \mathrm{~h}$ at room temperature. In addition, squares were treated in PBS alone for comparison. After pre-treatment, squares were washed 5 times with PBS prior to adding $2 \times 10^{6}$ HUVEC. Cells were incubated in a tissue culture incubator for $2 \mathrm{~h}$ with gentle shaking. Squares were then rinsed gently twice with PBS, and the cells were fixed with Karnovsky's fixative (2.5\% paraformaldehyde, $2 \%$ glutaraldehyde in $0.1 \mathrm{M}$ sodium phosphate buffer) for $2 \mathrm{~h}$. After two subsequent washes in PBS, cells were treated with $1 \% \mathrm{OsO}_{4}$ (Sigma-Aldrich) for $1 \mathrm{~h}$, followed by acetone dehydration. Cell images were obtained using an Environmental Scanning Electron Microscopy (ESEM) from the Department of Biology at Duke University.

\section{DNA Assay}

Total DNA content of HUVECs on each sample was measured as an index of cell number. PGA mesh pretreatment and cell seeding followed the same procedure as described above. After washing, PGA squares were digested with papain solution at $65^{\circ} \mathrm{C}$ overnight to remove cellular proteins. Samples were stained with Hoescht 33258 dye, and fluorescence was measured at $458 \mathrm{~nm}$ (excitation at $365 \mathrm{~nm}$ ) and compared to a standard curve to determine DNA concentration.

\section{Thin PGA Film Preparation}

Thin PGA films were prepared by spin-casting. PGA mesh was dissolved into hexafluoroisopropanol (HFIP, Sigma) to make 0.5\% (w/v) solution. QSX-301 standard gold sensor crystals (Q-Sense) were coated with $20 \mu \mathrm{L}$ of PGA solution. Glass coverslips $(22 \times 22 \mathrm{~mm})$ were coated with $40 \mu \mathrm{L}$ of PGA solution. Samples were spun at $3000 \mathrm{rpm}$ for $30 \mathrm{~s}$ and then dried at $80{ }^{\circ} \mathrm{C}$ for $30 \mathrm{~min}$. Spin-coated PGA thin films were stored in a dark and dry environment for short periods of time.

\section{Affinity Constant Measurement}

PGA-RGD-(7-1) peptide solutions in PBS were serially diluted to $0.034,0.17,0.85$, and $3.4 \mathrm{nmol} / \mathrm{mL}$ 
and all dilutions were degassed by sonication. PGA-coated QCM sensors were saturated with PBS until equilibrium was reached. The QCM-D D300 (Q-Sense Inc, Glen Bumie, MD) dynamic analysis system was set up to flow each solution continuously over the sensor surface at a speed of $1.25 \mathrm{~mL} / \mathrm{min}$. PGARGD-(7-1) peptide solutions were sequentially flowed through the sensor chamber, starting at the lowest concentration. When the equilibrium was established for one concentration, a solution with a higher peptide concentration was applied. After the solution with the highest peptide concentration reached equilibrium, the intake was switched to PBS to monitor peptide detachment from the PGA surface until a new equilibrium was reached. Peptide equilibrium thicknesses at different concentrations were calculated based on frequency and dissipation changes. The affinity constant was calculated from the concentrations of peptide solutions and their correlated equilibrium thicknesses using Q-Tools 2.0 modeling software (Q-Sense).

\section{Cell Spreading}

PGA spin-coated cover slips were treated with PBS, $20 \mu \mathrm{g} / \mathrm{mL}$ Fn in PBS, or $50 \mu \mathrm{g} / \mathrm{mL}$ PGA-RGD-(7-1) peptide in PBS for $1 \mathrm{~h}$ at room temperature. The surfaces were gently washed twice with excessive PBS and then transferred into 6-well tissue culture plates. $10^{4}$ HUVECs stained with Cell-tracker Green (Molecular Probes) and re-suspended in $2 \mathrm{~mL}$ serum free medium were injected into each well. The HUVECs were seeded for $30 \mathrm{~min}, 1 \mathrm{~h}, 2 \mathrm{~h}$, and $4 \mathrm{~h}$ at $37^{\circ} \mathrm{C}$. After incubation, cover slips were washed twice with PBS. Cells were fixed with $4 \%$ formaldehyde PBS solution for $20 \mathrm{~min}$. Fluorescent images were taken at five different fields for each cover slip, and the sizes of at least 30 individual cells were measured. Results were obtained from at least four replicate experiments.

\section{Actin and Vinculin Immunofluorescence}

Samples for actin and vinculin immunofluorescence were prepared following the procedure described above. After fixation, $0.2 \%$ Triton X-100 in PBS was used to permeablize cells for $5 \mathrm{~min}$. Cells were blocked with $3 \%$ BSA-PBS for $1 \mathrm{~h}$ at room temperature and then incubated with mouse anti-vinculin antibody (Sigma) for $1 \mathrm{~h}$, followed by washing twice for $5 \mathrm{~min}$ each. A solution containing both goat-anti-mouse-IgG secondary antibody conjugated with FITC (Chemicon) and rhodamine-phalloidin (Chemicon) was used to treat cells for $1 \mathrm{~h}$. Two 5-min PBS washes were applied. Images were taken at $100 \times$ total magnification.

\section{Statistical Analysis}

One-way analysis of variance (ANOVA) with Dunnett's or unpaired $t$-test was used when performing multiple comparisons as a post test (GraphPad Prism version 4.00, San Diego, CA). A value of $p<0.01$ was considered statistically significant. Data were expressed as mean \pm SEM.

\section{RESULTS}

\section{Isolation of Affinity Peptides for PGA}

In order to develop bio-adhesive coatings for PGA, we first sought to derive peptides that bound PGA with high affinity. We panned several peptide phage display libraries developed in our laboratory ${ }^{22,23,30}$ over PGA mesh as described in the methods. As part of our design, we chose libraries that displayed peptide sequence of $\leq 20$ amino acid, since longer peptides are more likely to be immunogenic. After three rounds of panning, a subpopulation of peptide phage was enriched with high affinity for PGA mesh as determined by phage pool ELISA (data not shown). Individual candidate clones were isolated for sequencing and analysis from rounds two and three. By the third round of panning, a single dominant clone had emerged (clone 7 in Fig. 2), thereby limiting our ability to derive useful sequence-activity information from other clones. In order to analyze a diversity of clones for structure-function relationships, round two clones were therefore selected for further characterization and determination of relative binding affinity using

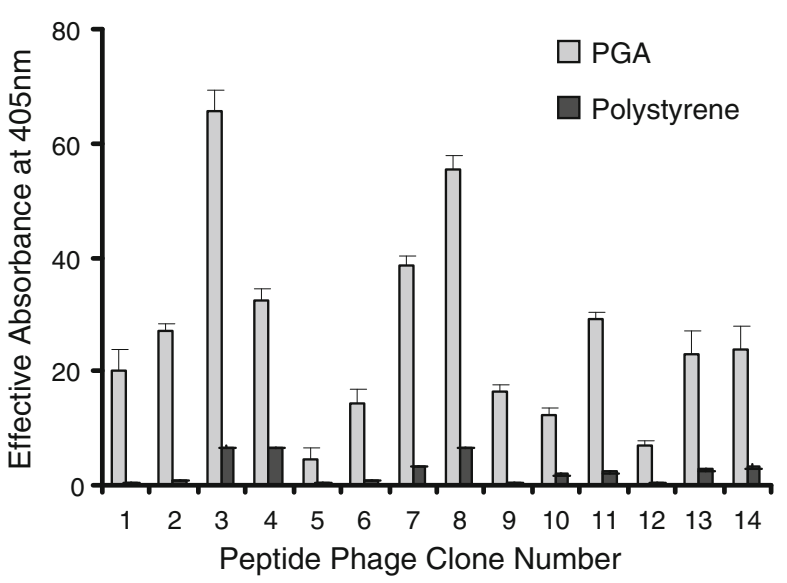

FIGURE 2. Representative ELISA values for peptide phage binding to PGA. Individual phage clones from PGA selection round 2 were amplified in liquid culture. After washing away unbound phage, HRP-conjugated anti-M13 antibodies were added, then incubated with the chromagen ABTS, and bound phage measured by absorbance at $405 \mathrm{~nm}(n>3)$. 
on-phage ELISA assay. As shown in Fig. 2 and Table 1, 45 out of 96 round two phage clones picked for screening displayed a high apparent affinity for PGA and a low affinity for polystyrene (used here as a negative control). Several of these clones were recovered more than once (Table 1), suggesting that their encoded peptides confer an enrichment advantage that may reflect high binding, a biological survival advantage, or both. Clone 7 was of particular interest, showing both high apparent binding to PGA and a high frequency of occurrence in the round 2 enriched population (18 of 96 clones picked for screening). Therefore, the sequence displayed by clone 7 was selected and subsequently incorporated into the IFBMs used for this study since it displayed a relative high affinity and occurrence within the screening population.

\section{Construction of Interfacial Biomaterials}

The sequence of PGA-binding clone 7 was incorporated into a synthetic peptide along with the tripeptide sequence RGD, known to recapitulate certain biological functions of the matrix proteins fibronectin and vitronectin, including integrin binding, focal adhesion kinase activation, and cell survival. ${ }^{13,16,32}$ The two functional modules were separated by a

TABLE 1. PGA-binding peptide sequences recovered by phage display, ranked according to their relative affinities by ELISA.

\begin{tabular}{llcc}
\hline Clone & \multicolumn{1}{c}{ Amino acid sequences } & Relative affinity & Occurrence \\
\hline 3 & SCSFFMPWCNFLNGEMAVS & 65.5 & 1 \\
8 & SCGYFCSFYNYLDIGTASS & 55.3 & 1 \\
7 & SCNSSSYSWYCWFGGSSPS & 38.7 & 18 \\
4 & SCFGNVFCVYNQFAAGLFS & 32.3 & 1 \\
11 & SCFGFSDCLSWFVQPSTAS & 29.1 & 1 \\
2 & SCFGNLGNLIYTCDRLMPS & 27.2 & 3 \\
14 & SCWMGLFECPDAWLHDWDS & 23.7 & 1 \\
13 & SCNHLGFFSSFCDRLVENS & 23.0 & 1 \\
1 & SCNSFMFINGSFKETGGCS & 20.0 & 9 \\
9 & SCFGNFLSFGFNCESALGS & 16.2 & 1 \\
6 & SCDYFSFLECFSNGWSGAS & 14.4 & 1 \\
10 & SCLYCHLNNQFLSWVSGS & 12.4 & 3 \\
12 & SCCFINSNFSVMNHSLFKS & 7.1 & 3 \\
5 & SCFWYSWLCSASSSDALIS & 4.4 & 1 \\
\hline
\end{tabular}

Occurrence indicates the number of times each clone was recovered within 45 representative phage clones in the PGA-enriched pool. negatively charged five amino acid linker sequence, Ala-Glu-Ala-Glu-Ala (AEAEA), chosen to provide spacing and sufficient charge to assist with peptide solubility without imparting a strong structural influence on the short peptide. The resulting peptide, termed PGA-RGD-(7-1), is shown in Table 2, along with a negative control peptide in which the RGD moiety is replaced by RGE, termed PGA-RGE-(7-2), known not to bind to integrin receptors. ${ }^{6}$ The domain organization of an IFBM is illustrated schematically in Fig. 1a. The hypothesized use of the PGA-RGD IFBM to modify PGA surfaces to mediate endothelial cell interactions is illustrated in Fig. 1 b.

\section{Biophysical Characterization of IFBM Binding to PGA}

Surface thicknesses of IFBM peptide PGA-RGD(7-1) coatings under static conditions were determined by QCM-D over a range of peptide concentrations (Fig. 3a). For these experiments, a PGA coated crystal sensor was used, instead of the PGA meshes. The dissipation changes after peptide adsorption were all under $1 \times 10^{-6}$, which indicated that the peptide coating behaved as a thin and rigid coating and that viscoelastic effects were minimal. Both Sauerbrey and Voigt modeling ${ }^{15}$ gave virtually identical calculations of thickness (Fig. 3a), which further supported the interpretation of a thin and rigid coating. These data indicate that surface saturation occurs at a concentration of approximately $10 \mu \mathrm{g} / \mathrm{mL}$ and that the coating reaches a maximum thickness at saturation of approximately $3.5 \mathrm{~nm}$. Thus, the PGA surface saturated with PGA-RGD-(7-1) peptide has a peptide density of $\approx 0.12 \mathrm{nmol} / \mathrm{cm}^{2}$. For comparison, the thickness of the Fn layer on a saturated PGA surface, which was saturated with $20 \mu \mathrm{g} / \mathrm{mL}$ Fn PBS solution, was also measured by QCM-D under static condition. The thickness of the Fn layer was approximately $8.5 \pm 0.2 \mathrm{~nm}$, which represents a Fn surface density of $\approx 0.002 \mathrm{nmol} / \mathrm{cm}^{2}$.

Kinetic measurements were conducted by sequentially flowing different concentrations, from low to high, of PGA-RGD-(7-1) peptide solutions over the PGA coated crystal sensor. When the equilibrium between the solution and surface was reached, a higher concentration of peptide solution was applied.

TABLE 2. Peptide sequences for the interfacial biomaterial constructs synthesized and used in this study.

\begin{tabular}{llll}
\hline Designation & \multicolumn{1}{c}{ PGA-binding domain } & Linker & Biologic-binding domain \\
\hline PGA-RGD-(7-1) & SCNSSSYSWYCWFGGSSPS & AEAEA & \multicolumn{1}{c}{ RGD } \\
PGA-RGE-(7-2) & SCNSSSYSWYCWFGGSSPS & AEAEA & RGE \\
PGA-bio-(7-3) & SCNSSSYSWYCWFGGSSPS & AEAEA & K-biotin \\
\hline
\end{tabular}


(a)
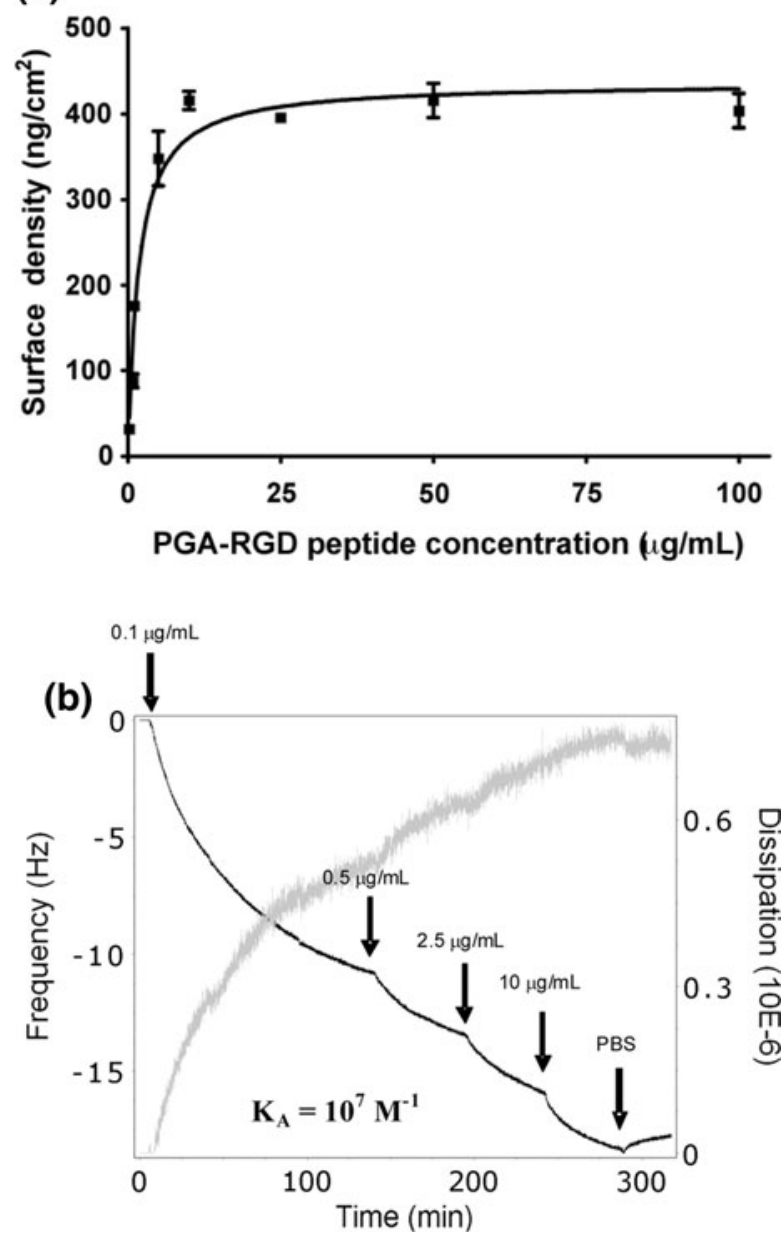

FIGURE 3. Representative QCM-D characterization of IFBM binding to PGA. (a) Saturation curve of peptide PGA-RGD(7-1) thickness after binding to PGA-coated sensor chips. QCM-D was used to determine surface thickness under static conditions by both Sauerbrey and Voigt modeling over a range of peptide concentrations. Both models gave essentially identical thicknesses at each point, and their data points are superimposed. (b) QCM-D measurements of IFBM PGARGD-(7-1) binding to PGA-coated sensor chips. Frequency and dissipation were measured during continuous flow over time. The first four arrows from the left correspond to addition of increasing amounts of peptides to the flow solution (0.1, $0.5,2.5$, and $10 \mu \mathrm{g} / \mathrm{mL}$, respectively). The last arrow corresponds to initiation of washing in buffer without peptide added. The association constant $K_{\mathrm{A}}$ was calculated as described in the Methods.

When the surface was saturated, PBS was applied to wash the PGA surface. The frequency and dissipation changes were monitored during the entire process (Fig. 3b), and were similar to data measured under static conditions. The saturation thickness measured under kinetic conditions (Table 3) was slightly smaller than, but still in line with, the measurements under static condition. The affinity constant $K_{\mathrm{A}}$ was calculated according to the frequency drops and dissipation
TABLE 3. Kinetic modeling results for PGA-RGD-(7-1) peptide obtained form the QCMD experiments.

\begin{tabular}{lccc}
\hline $\begin{array}{l}\text { Concentration } \\
(\mu \mathrm{g} / \mathrm{mL})\end{array}$ & $\begin{array}{c}\text { Concentration } \\
(\mathrm{nmol} / \mathrm{mL})\end{array}$ & $\begin{array}{c}\text { Sauerbrey } \\
\text { thickness }(\mathrm{nm})\end{array}$ & $\begin{array}{c}\text { Voigt } \\
\text { thickness }(\mathrm{nm})\end{array}$ \\
\hline 0.1 & 0.034 & $1.6 \pm 0.3$ & $1.6 \pm 0.3$ \\
0.5 & 0.17 & $2.1 \pm 0.4$ & $2.1 \pm 0.4$ \\
2.5 & 0.85 & $2.6 \pm 0.2$ & $2.6 \pm 0.2$ \\
10 & 3.4 & $3.0 \pm 0.2$ & $2.9 \pm 0.2$ \\
\hline
\end{tabular}

increases in the kinetic measurements. In brief, a straight line plot of $C / R_{\text {eq }}$ vs. $C$ had a slope of $1 / R_{\max }$ and a $\mathrm{Y}$ intercept of $1 / K_{\mathrm{A}} R_{\max }$, where $C$ was the concentration of IFBM, $R_{\mathrm{eq}}$ was the equilibrium QCM-D response, $R_{\max }$ was the maximal QCM-D response, and $K_{\mathrm{A}}$ was the kinetic association constant (see resultant data in Table 3). $R_{\max }$ calculated by both Sauerbrey and Voigt modeling was in close agreement over the range of peptide concentrations, yielding a $K_{\mathrm{A}}$ of $1 \times 10^{7} \mathrm{M}^{-1}$, which is approximately 10 -fold higher than the affinity constant reported for Fn with its receptor $\alpha_{5} \beta_{1}{ }^{1}$

\section{PGA-RGD IFBMs Mediate Endothelial Cell Binding to PGA Mesh}

HUVEC were plated onto PGA mesh pre-treated with either buffer alone, IFBM PGA-RGD-(7-1), or control peptide PGA-RGE-(7-2) and the cells allowed to bind for $2 \mathrm{~h}$ prior to washing with PBS. For these experiments, a stock solution containing a known amount of cells was used for the assay. Aliquots were taken from the stock and the stock solution was gently rocked between the aliquots to ensure that approximately the same number of cells were used in every well. We also used a green/red (live/dead) stain to confirm cell viability. After washing, cells were prepared for ESEM imaging or DNA content measurement. Images of the PGA mesh show that neither PGA alone nor PGA treated with control peptide PGARGE-(7-2) bound significant numbers of HUVECs (Figs. 4a and 4c), which is in agreement with the DNA content determinations. As shown in Fig. 4e, the DNA content of HUVEC plated onto PGA mesh alone or PGA treated with control peptide PGA-RGE-(7-2) is low, indicating poor cell binding. In contrast, PGA mesh treated with IFBM PGA-RGD-(7-1) binds a larger number of HUVECs (Fig. 4b). The DNA content of cells plated onto PGA treated with the IFBM PGA-RGD-(7-1) is 3 times higher. Similar results were obtained by Western blotting assay (data not shown). Higher magnification reveals that the IFBM PGA-RGD-(7-1) treated PGA fibers are encased by HUVECs (Fig. 4d). 

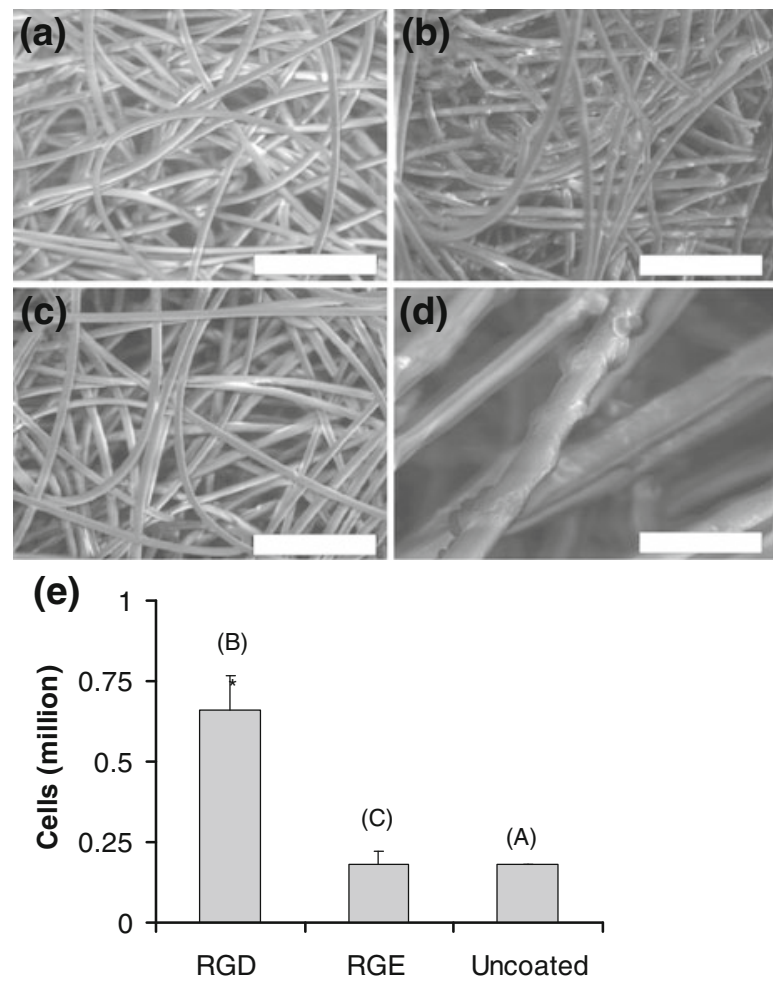

FIGURE 4. IFBM-mediated binding of endothelial cells to PGA fibers. A single cell suspension of cultured HUVEC containing $2 \times 10^{6}$ cells was applied to a nonwoven PGA mesh $1 \mathrm{~cm}^{2}$ in size pretreated with (a) buffer only; (b) buffer plus IFBM PGA-RGD-(7-1); or (c) buffer plus PGA-RGE-(7-2). (d) A higher magnification view of panel (b). Samples were freeze-dried and visualized by SEM. Size bars are $200 \mu \mathrm{m}$ in panels a-c and $20 \mu \mathrm{m}$ in panel d. (e) DNA content was assayed after addition of Hoescht 33258 dye solution and related to cell number by comparison to a standard curve $(p<0.01)$.

\section{Effects of IFBM PGA-RGD on HUVEC Function}

In order to better visualize the morphology of cultured cells on PGA, we spin-coated PGA onto glass coverslips for subsequent use in cell culture and microscopy. We also compared the behavior of endothelial cells plated on our IFBM to that of cells plated onto the prototypical RGD-containing matrix protein, Fn. Neither PBS nor the control peptide PGARGE-(7-2) was able to mediate significant HUVEC adhesion or spreading on PGA surfaces. In contrast, both IFBM PGA-RGD-(7-1) and Fn were able to mediate significant cell adhesion and spreading, as expected. These images were observed by fluorescence microscopy and the cell area quantified at the 4-h time point (Fig. 5). These data indicate that the IFBM PGA-RGD-(7-1) and Fn both induced significantly more cell spreading compared to bare PGA. Moreover, there were no significant differences evident between Fn and the IFBM peptide, indicating that both coatings can provide sufficient cues for function. The potential advantages of using the IFBM peptide,

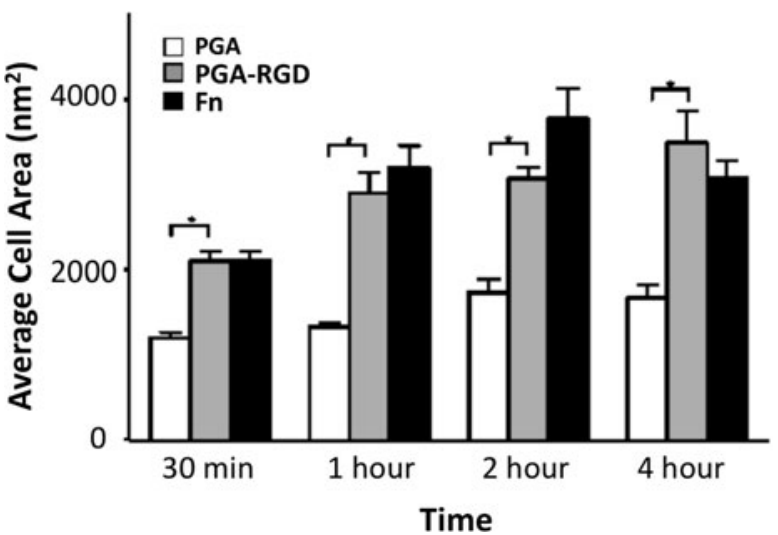

FIGURE 5. Time course of HUVEC spreading on PGA surfaces. PGA spin-coated coverslips were prepared and left either uncoated (PGA), coated with IFBM PGA-RGD-(7-1) (RGD), or coated with fibronectin. HUVECs were treated with Cell-tracker Green, plated and allowed to adhere and spread for the indicated times, then fixed and measured by fluorescence microscopy $(p<0.01)$.

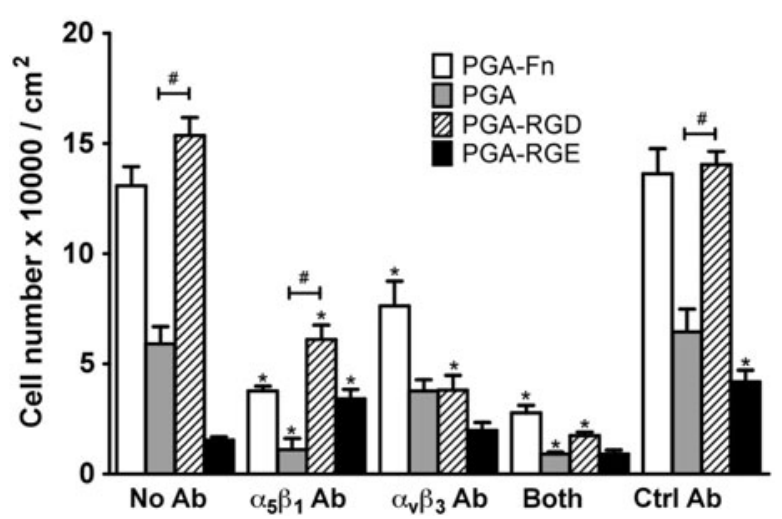

FIGURE 6. HUVECs were re-suspended in five different solutions: PBS, PBS with $10 \mu \mathrm{g} / \mathrm{mL}$ anti- $\alpha_{v} \beta_{3}$ integrin antibodies, PBS with $10 \mu \mathrm{g} / \mathrm{mL}$ anti- $\alpha_{5} \beta_{1}$ integrin antibodies, PBS with $10 \mu \mathrm{g} / \mathrm{mL}$ of both antibodies, and PBS with $10 \mu \mathrm{g} / \mathrm{mL}$ of control Ig $\mathrm{G}_{1}$ antibodies. Cells then were cultured in these PBS antibody solutions for $1 \mathrm{~h}$ at room temperature with gentle shaking, and then were seeded onto four different preprepared surfaces for $30 \mathrm{~min}$. Five fluorescent images at random fields on each slide were taken for analysis with four repeats. Each of anti- $\alpha_{5} \beta_{1}$ and anti- $\alpha_{v} \beta_{3}$ antibodies individually blocked HUVECs adhesion on both Fn- and PGA-RGD-coated surfaces. Adhesion inhibition increased further when both antibodies were applied together. No statistical difference was observed in HUVECs adhesion on PGA and PGA-RGD surfaces after cells were treated with anti- $\alpha_{v} \beta_{3}$ antibody. ${ }^{\#} p<0.01 ;{ }^{*} p<0.001$.

over Fn, include the ability to precisely control the type and number of interactions in a well-defined, chemically synthesized coating.

Integrin receptor blocking assays were conducted to further evaluate the roles of RGD-integrin interactions in cell adhesion onto PGA-RGD-(7-1) modified PGA surfaces (Fig. 6). Two cell membrane integrin receptors, $\alpha_{v} \beta_{3}$ and $\alpha_{5} \beta_{1}$ were studied. Anti- $\alpha_{\mathrm{v}} \beta_{3}$ 

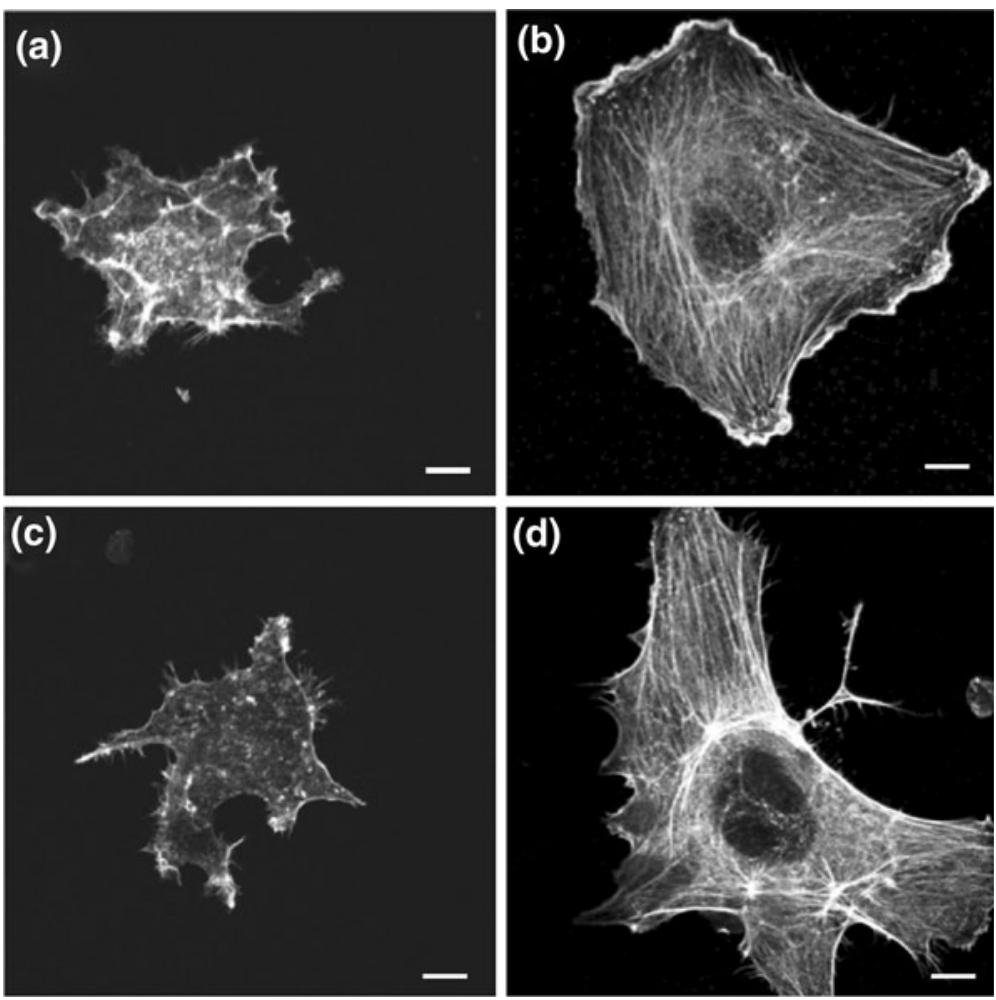

FIGURE 7. Confocal microscope images of HUVECs fluorescently stained for actin fibers. Endothelial cells seeded onto (a) noncoated PGA, (b) PGA-RGD-(7-1) coated; (c) PGA-RGE-(7-2) coated; and (d) Fn-coated surfaces were fixed and stained after $1 \mathrm{~h}$ and observed at $100 \times$ magnification. Scale bars are $10 \mu \mathrm{m}$. HUVECs plated onto either PGA or control peptide PGA-RGE-(7-2) did not show appreciable actin fiber organization after $1 \mathrm{~h}$ cell seeding $(\mathrm{a}, \mathrm{c})$. In contrast, ECs on both IFBM PGA-RGD-(7-1) and Fn showed dramatic actin fiber organization $(b, d)$.

antibody and anti- $\alpha_{5} \beta_{1}$ antibody were used to treat EC individually and collectively. A control antibody with the same $\alpha$ and $\beta$ chains was used as a negative control. The results showed that both integrin receptors participated in the recognition of IFBM and Fn, and that no other cellular interactions with the IFBM peptide played a role in cellular adhesion. Interestingly, the antibodies were not able to fully block binding to Fn, consistent with the fact that Fn also interacts with cells via additional receptors. ${ }^{40,50}$

Upon integrin binding by extracellular fibronectin, the integrin intracellular domains participate in the formation of focal adhesion plaques involving the actin cytoskeleton and certain adaptor proteins such as vinculin. ${ }^{11,53}$ We therefore compared the distribution of actin and vinculin following exposure of endothelial cells to PGA surfaces with different coatings. As expected, endothelial cells plated onto either PGA or control peptide PGA-RGE-(7-2) did not show appreciable actin fiber organization or redistribution of vinculin into focal adhesion plaques after $1 \mathrm{~h}$ cell seeding (Figs. 7a, 7c and 8a). In contrast, ECs on both IFBM PGA-RGD-(7-1) and Fn showed dramatic actin fiber organization (Figs. $7 \mathrm{~b}$ and $7 \mathrm{~d}$ ) and started to show vinculin redistribution into a predominantly perinuclear focal adhesion plaque pattern (Figs. 8b and $8 \mathrm{c}$ ). Cells showed clearer vinculin plaques after $2 \mathrm{~h}$ of seeding onto PGA-RGD-(7-1) and Fn surfaces (Figs. 8e and 8f).

\section{DISCUSSION}

We are designing peptide coatings that function at the interface to control biological responses on inert surfaces such as metals and plastics. ${ }^{23,24,34-36,54}$ These bifunctional, two-domain peptide coatings must recognize and bind both the inert surface through non-covalent physisorption, while also providing a mechanism to interact with the surrounding biology. In this fashion, these peptides create an interface that allows for appropriate biological interactions with inert materials. Our previous research focused on using bifunctional resulting peptides to regulate the interactions that occurred at the biological-material interface including: promotion of surface cellularization, ${ }^{34}$ suppression of apoptosis, ${ }^{36}$ prevention of fouling, ${ }^{23,24}$ and, more recently, release of a therapeutic from a 

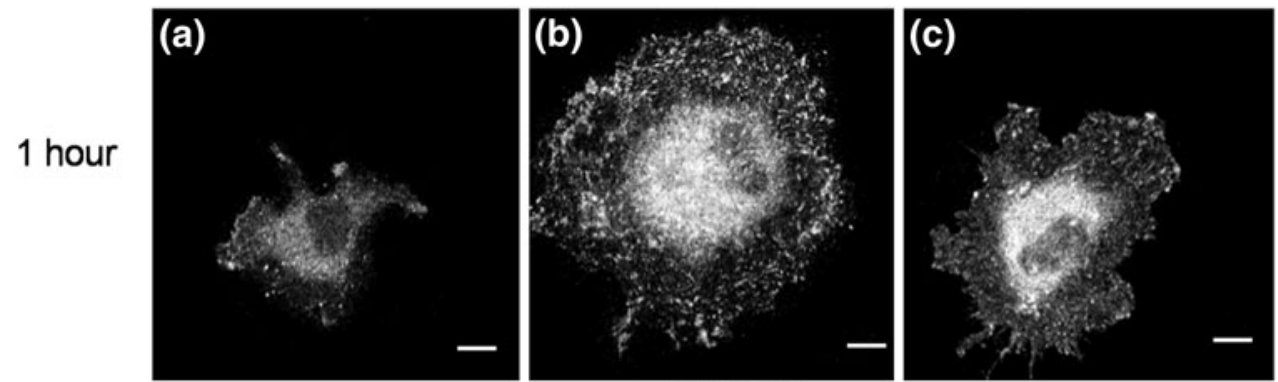

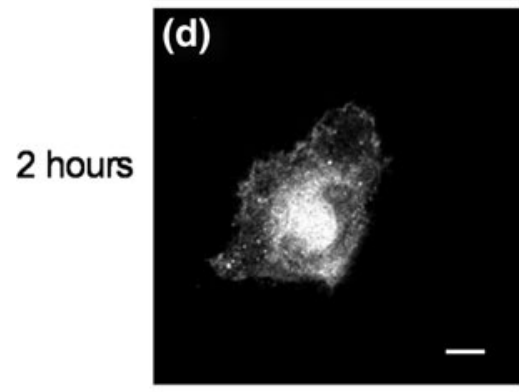

PGA

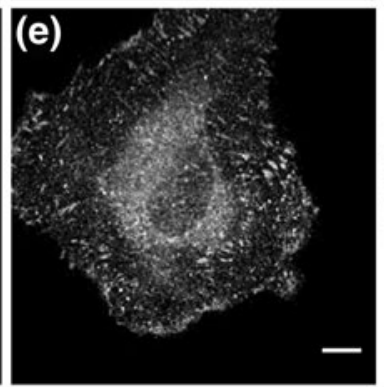

PGA-RGD-(7-1)

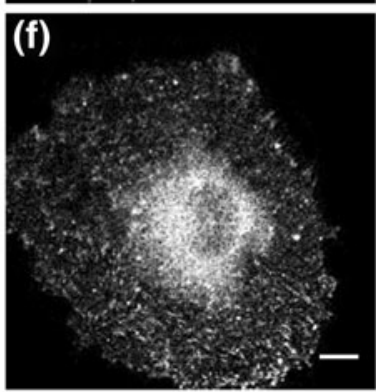

PGA-Fn

FIGURE 8. Confocal microscope images of HUVECs fluorescently stained for vinculin. Endothelial cells seeded onto (a) noncoated PGA, (b) PGA-RGD-(7-1) coated; (c) Fn coated PGA surfaces after 1 h; (d) non-coated PGA, (e) PGA-RGD-(7-1) coated; (f) Fn coated PGA surfaces after $2 \mathrm{~h}$ and observed at 100x magnification. Scale bars are $10 \mu \mathrm{m}$. HUVECs plated onto either PGA or control peptide PGA-RGE-(7-2) did not show appreciable redistribution of vinculin into focal adhesion plaques after $1 \mathrm{~h}$ cell seeding (a). In contrast, ECs on both IFBM PGA-RGD-(7-1) and Fn started to show dramatic vinculin redistribution into a predominantly perinuclear focal adhesion plaque pattern $(b, c)$. Cells showed clearer vinculin plaques after $2 \mathrm{~h}$ of seeding onto PGA-RGD-(7-1) and Fn surfaces (e, f).

surface. ${ }^{35}$ In this report, we have approached the problem of poor biological activity of PGA fibers by developing a modular IFBM peptide that is capable of binding to PGA and modifying its surface to provide biological cues that direct endothelial cell adhesion, spreading, cytoskeletal reorganization, and focal adhesion plaque formation.

Phage display was used in this study to develop modular binding domains with strong affinity to PGA surfaces. Phage display is a powerful selection tool which can be used to select peptides with desired properties from vast peptide libraries, typically exceeding $10^{9}$ variant peptide clones. Smith and colleagues first described the phage display method in $1985,{ }^{49}$ and this technique has been widely applied since. Most phage display applications have been to study interactions between biological molecules, both in vivo and in vitro. ${ }^{19,21}$ Phage display has also been applied to derive peptides with affinity for inorganic or biomaterial surfaces including metals, metal oxides, semiconductors, carbon nanotubes, and polymeric materials. ${ }^{27} \mathrm{We}$ have also previously reported peptides that bind metals, metal oxides, and plastics. ${ }^{23,34,54}$ Both Sanghvi et al. and Meyer et al. individually described bimodular RGD peptides that promoted cell adhesion to polypyrrole and $\mathrm{Ti}$ metal, respectively. ${ }^{34,46,47}$ Application of IFBMs to degradable biomaterials such as PGA has not been previously reported.

We used a quartz crystal microbalance with dissipation monitoring (QCM-D) to study molecular interactions and affinity constants. ${ }^{31}$ Our data show that binding of IFBM to the spin-coated PGA surface is rapid and efficient. Furthermore, the IFBM PGARGD-(7-1) adsorbed as a monolayer on the PGA surface, and the adsorption exhibited isothermal, Langmuir-like behavior. The measured affinity constant, $10^{7} \mathrm{M}^{-1}$, agreed with the measurements from biotin ELISA assays (data not shown). Assuming that the IFBM peptide forms a random-coil structure with $0.35 \AA$ per amino acid, this peptide is estimated to be $9.5 \mathrm{~nm}$ in length. The layer thickness at monolayer coverage is about $3.5 \mathrm{~nm}$ determined from Sauerbrey model. This thickness suggests that at least a portion of the peptide length extends into the solution phase above the surface. Given the charged nature of the RGD domain, the polarity of the linker, and the demonstrated ability of the bound IFBMs to mediate cell binding via integrin receptors, we conclude that these IFBMs self-orient onto the PGA surface with the PGAbinding domain oriented down toward the surface and the RGD domain oriented toward the solution.

We also confirmed that integrin binding is responsible for the binding and spreading behavior exhibited 
by endothelial cells growing on PGA-RGD IFBM coated surfaces. Our studies show that competition with soluble RGD tripeptide effectively blocks the ability of PGA-RGD IFBMs to mediate HUVEC binding to coated PGA surfaces (data not shown). We definitively identified the integrin receptors involved by performing antibody competition experiments. Figure 6 shows that antibodies that bind either of the two major endothelial cell integrin receptors $\alpha_{5} \beta_{1}$ and $\alpha_{v} \beta_{3}$, each block approximately $50-70 \%$ of HUVEC binding to either Fn- or IFBM-coated surfaces. When both antibodies are used together in the competition, the inhibition approaches background binding levels for the IFBM, confirming that the IFBM PGA-RGD(7-1) binds HUVEC through integrin receptors. Interestingly, antibody competitions on Fn-coated surfaces never inhibit HUVEC binding to as high a degree (Fig. 6), consistent with the fact that Fn can mediate cellular interactions via receptors other than the RGD-binding integrins as reported previously. ${ }^{40,50}$ Integrin mediated endothelial cell adhesion also activates cell focal adhesion pathways. Thus, we investigated the formation and rearrangement of actin fibers and vinculin plaque inside endothelial cells on these three different surfaces over a period of $4 \mathrm{~h}$. Endothelial cells formed organized actin fibers and vinculin plaques $1 \mathrm{~h}$ after incubation on both Fn and IFBM PGA-RGD-(7-1) surfaces in serum free medium, but not on uncoated PGA surfaces even after $4 \mathrm{~h}$ of incubation. These findings further demonstrate that our IFBM peptide modifies PGA surfaces to recapitulate key elements of ECM structure and function.

We note that, in comparison to the approach presented here, previous surface RGD modification methods showed considerable disadvantages. ${ }^{13}$ For example, it was shown that simple adsorption of small RGD molecules does not lead to cell attachment. ${ }^{7}$ Two popular strategies for RGD immobilization, derivatization of polymers to introduce RGD and immobilization of RGD peptides on polymer surfaces, attempt to overcome this obstacle. The polymer derivatization methods include blending, co-polymerization, networking, and chemical/physical treatments. ${ }^{2,42}$ These methods typically require multiple steps of chemical treatment and reactions under specific conditions that complicate the RGD-introduction process and limit its applications to select materials. Immobilization of RGD peptides onto biomaterials has also been broadly studied, and some products have even been commercialized. For example, poly-L-lysine (PLL) has been reported to be conjugated with GRGDS to promote cell adhesion onto synthetic polymer surfaces. ${ }^{41}$ PLL was reported to show a high degree of cell toxicity when its concentration was above $0.5 \mu \mathrm{g} / \mathrm{cm}^{2} .{ }^{51}$ Hexaleucine conjugated with GRGDS was also studied to improve cell adhesion (PepTite-2000 $\left.{ }^{\mathrm{TM}}\right){ }^{8}$ But other research showed this PepTite product causes an unexpected plasma protein deposition. ${ }^{38}$ With respect to modification of PGA surface, hydrolysis of the ester linkages followed by chemical treatment to afford an activated ester (e.g., PFF) has been used to introduce amine terminated RGD peptides in specific patterns. ${ }^{29}$ Alternatively, a group has prepared a PGLA-PEG- $\mathrm{NH}_{2}$ block polymer and incorporated this diblock polymer into the PGLA for subsequent chemical coupling to an RGD peptide. ${ }^{25}$ More recently, an ultra low molecular weight functionalized PLGA has been prepared that possesses an acrylated integrin-binding Ac-GRGD amino acid sequence which could then be polymerized into a scaffold. ${ }^{17}$ In a similar approach, photocrosslinkable PEG-PGA blocks have been prepared and subsequently used to create scaffold containing RGD. ${ }^{4}$ The IFBM peptide developed by our group represents a simple and robust treatment that overcomes the aforementioned disadvantages. The peptides are applied by a simple dip coat in aqueous solution, and the modularity of the IFBMs assures that different materials and biologics can be targeted simply by changing the terminal peptide domains. Moreover, the economics of large scale peptide synthesis indicate that coatings can be applied to polymers and polymer scaffolds for pennies to a few dollars per device, depending on the size of the device and the complexity of the coating.

In our first study there are two limitations: (1) the experiments were performed under static conditions and (2) the use of the RGD motif for binding to endothelial cells. Flow and shear can have a significant effect on cell adhesion and spreading as well as other biological functions. In a separate study, using an IFBM that mediated the interaction between cells and a Ti surface, we did observe an even more pronounced effect (e.g., spreading) under flow conditions. ${ }^{34} \mathrm{We}$ selected the RGD motif because it is the classic and most widely studied integrin receptor binder, and also because it has been used successfully as a key element in surface coatings. ${ }^{3,13,32,39,45}$ However, it is also ubiquitous and the sequence binds approximately half of all known human integrins, including the integrin on both endothelial cells and platelets. An additional caveat to the above study is that the IFBM binding experiments were conducted on a PGA spin coated surface and the cell binding studies were performed on a PGA fiber mesh. It is known that increased surface roughness can also influence cell binding and spreading. ${ }^{28}$ However, these experiments are an important first step in the evaluation of this new coating for PGA meshes, and the modularity of the IFBM approach will enable the replacement of the RGD with a suitable peptide. 
In summary, we have successfully developed a short peptide that is able to easily, quickly, and effectively modify PGA surfaces to promote endothelial cell adhesion and spreading. These results are of particular interest to PGA-based scaffolds used in artificial vascular engineering where endothelial cell function is critical for device performance. Further studies are underway to examine the ability of this peptide to retain cells under shear stress as well as to direct other EC biological functions. Given the modularity of the IFMB concept, it will be possible to create additional IFBMs with specific functions for a range of polymeric or metallic implantable devices where controlling the biologic-material interface is critical for optimal performance.

\section{ACKNOWLEDGMENTS}

This work was supported by the National Institutes of Health (Grant 5R01EB000501 to MWG) and the North Carolina Biotechnology Center (Collaborative Funding Grant to DJK supporting XH). The authors thank Felix Yap for assistance with image analysis, and Erin Carruthers, PhD, for assistance with QCM-D.

\section{REFERENCES}

${ }^{1}$ Akiyama, S. K., and K. M. Yamada. The interaction of plasma fibronectin with fibroblastic cells in suspension. J. Biol. Chem. 260:4492-4500, 1985.

${ }^{2}$ Anderson, J. M. Biological responses to materials. Annu. Rev. Mater. Res. 31:81-110, 2001.

${ }^{3}$ Barber, T. A., J. E. Ho, A. De Ranieri, A. S. Virdi, D. R. Sumner, and K. E. Healy. Peri-implant bone formation and implant integration strength of peptide-modified $\mathrm{p}(\mathrm{aam}-\mathrm{co}-\mathrm{eg} / \mathrm{aac})$ interpenetrating polymer networkcoated titanium implants. J. Biomed. Mater. Res. A 80: 306-320, 2007.

${ }^{4}$ Bencherif, S. A., A. Srinivasan, J. A. Sheehan, L. M. Walker, C. Gayathri, R. Gil, J. O. Hollinger, K. Matyjaszewski, and N. R. Washburn. End-group effects on the properties of peg-co-pga hydrogels. Acta Biomater. 5:1872-1883, 2009.

${ }^{5}$ Chen, R., and J. A. Hunt. Biomimetic materials processing for tissue-engineering processes. J. Mater. Res. 17:3974-3979, 2007.

${ }^{6}$ Cherny, R. C., M. A. Honan, and P. Thiagarajan. Sitedirected mutagenesis of the arginine-glycine-aspartic acid in vitronectin abolishes cell adhesion. J. Biol. Chem. 268:9725-9729, 1993.

${ }^{7}$ Cook, A. D., J. S. Hrkach, N. N. Gao, I. M. Johnson, U. B. Pajvani, S. M. Cannizzaro, and R. Langer. Characterization and development of rgd-peptide-modified poly(lactic acid-co-lysine) as an interactive, resorbable biomaterial. J. Biomed. Mater. Res. 35:513-523, 1997.
${ }^{8}$ Craig, W. S., S. Cheng, D. G. Mullen, J. Blevitt, and M. D. Pierschbacher. Concept and progress in the development of rgd-containing peptide pharmaceuticals. Biopolymers 37:157-175, 1995 .

${ }^{9}$ Day, R. M., A. R. Boccaccini, S. Shurey, J. A. Roether, A. Forbes, L. L. Hench, and S. M. Gabe. Assessment of polyglycolic acid mesh and bioactive glass for soft-tissue engineering scaffolds. Biomaterials 25:5857-5866, 2004.

${ }^{10}$ Garcia, A. J. Interfaces to control cell-biomaterial adhesive interactions. In: Polymers for Regenerative Medicine, edited by C. Werner. Springer, 2006, pp. 171-190.

${ }^{11}$ Geiger, B., A. Bershadsky, R. Pankov, and K. M. Yamada. Transmembrane crosstalk between the extracellular matrix - cytoskeleton crosstalk. Nat. Rev. Mol. Cell Biol. 2:793-805, 2001.

${ }^{12}$ Healy, K. E. Molecular engineering of materials for bioreactivity. Curr. Opin. Solid State Mater. Sci. 4:381-387, 1999.

${ }^{13}$ Hersel, U., C. Dahmen, and H. Kessler. Rgd modified polymers: biomaterials for stimulated cell adhesion and beyond. Biomaterials 24:4385-4415, 2003.

${ }^{14}$ Heydarkhan-Hagvall, S., M. Esguerra, G. Helenius, R. Soderberg, B. R. Johansson, and B. Risberg. Production of extracellular matrix components in tissue-engineered blood vessels. Tissue Eng. 12:831-842, 2006.

${ }^{15}$ Hook, F., B. Kasemo, T. Nylander, C. Fant, K. Sott, and $\mathrm{H}$. Elwing. Variations in coupled water, viscoelastic properties, and film thickness of a mefp-1 protein film during adsorption and cross-linking: a quartz crystal microbalance with dissipation monitoring, ellipsometry, and surface plasmon resonance study. Anal. Chem. 73:5796-5804, 2001.

${ }^{16}$ Hubbell, J. A. Biomaterials in tissue engineering. Biotechnology 13:565-576, 1995.

${ }^{17}$ Jabbari, E., X. He, M. T. Valarmathi, A. S. Sarvestani, and W. Xu. Material properties and bone marrow stromal cells response to in situ crosslinkable rgd-functionalized lactide-co-glycolide scaffolds. J. Biomed. Mater. Res. A 89: 124-137, 2009

${ }^{18}$ Jordan, S. W., and E. L. Chaikof. Novel thromboresistant materials. J. Vasc. Surg. 45:104A-115A, 2007.

${ }^{19}$ Kay, B. K., A. V. Kurakin, and R. Hyde-Deruyscher. From peptides to drugs via phage display. Drug Discovery Today 3:370-378, 1998.

${ }^{20}$ Kay, B. K., J. Winter, and J. Mccafferty. Phage Display of Peptides and Proteins. San Diego: Academic Press, 1996.

${ }^{21}$ Kehoe, J. W., and B. K. Kay. Filamentous phage display in the new millennium. Chem. Rev. 105:4056-4072, 2005.

${ }^{22}$ Kenan, D. J., W. J. Strittmatter, and J. R. Burke. Phage display screening for peptides that inhibit polyglutamine aggregation. Methods Enzymol. 413:253-273, 2006.

${ }^{23}$ Kenan, D. J., E. B. Walsh, S. R. Meyers, G. A. O'toole, E. G. Carruthers, W. K. Lee, S. Zauscher, C. A. H. Prata, and M. W. Grinstaff. Peptide-peg amphiphiles as cytophobic coatings for mammalian and bacterial cells. Chem. Biol. 13:695-700, 2006.

${ }^{24}$ Khoo, X., P. Hamilton, G. A. O’toole, B. D. Snyder, D. J. Kenan, and M. W. Grinstaff. Directed assembly of pegylated-peptides for infection-resistant titanium implant coatings. J. Am. Chem. Soc. 131:10992-10997, 2009.

${ }^{25}$ Kim, T. G., and T. G. Park. Biomimicking extracellular matrix: cell adhesive rgd peptide modified electrospun poly(d, l-lactic-co-glycolic acid) nanofiber mesh. Tissue Eng. 12:221-233, 2006.

${ }^{26}$ Kohn, J., and R. Langer. Bioresorbable and bioerodible materials. In: An Introduction to Materials in Medicine, 
edited by B. D. Ratner, A. S. Hoffman, F. J. Schoen, and J. E. Lemon. San Diego: Academic Press, 1997, pp. 65-73.

${ }^{27}$ Kriplani, U., and B. K. Kay. Selecting peptides for use in nanoscale materials using phage-displayed combinatorial peptide libraries. Curr. Opin. Biotechnol. 16:470-475, 2005.

${ }^{28}$ Lampin, M., R. Warocquier-Clérout, C. Legris, M. Degrange, and M. F. Sigot-Luizard. Correlation between substratum roughness and wettability, cell adhesion, and cell migration. J. Biomed. Mater. Res. A 36:99108,1998

${ }^{29}$ Lee, K. B., D. J. Kim, Z. W. Lee, S. I. Woo, and I. S. Choi. Pattern generation of biological ligands on a biodegradable poly(glycolic acid) film. Langmuir 20:2531-2535, 2004.

${ }^{30} \mathrm{Li}$, H., T. H. Labean, and D. J. Kenan. Single-chain antibodies against DNA aptamers for use as adapter molecules on DNA tile arrays in nanoscale materials organization. Org. Biomol. Chem. 4:3420-3426, 2006.

${ }^{31}$ Liu, Y., X. Yu, R. Zhao, D. H. Shangguan, Z. Bo, and G. Liu. Real time kinetic analysis of the interaction between immunoglobulin $\mathrm{g}$ and histidine using quartz crystal microbalance biosensor in solution. Biosens. Bioelectron. 18:1419-1427, 2003.

${ }^{32}$ Lutolf, M. P., and J. A. Hubbell. Synthetic biomaterials as instructive extracellular microenvironments for morphogenesis in tissue engineering. Nat. Biotechnol. 23:47-55, 2005.

${ }^{33}$ Mclarty, A. J., M. R. Phillips, D. R. Holmes, Jr., and H. V. Schaff. Aortocoronary bypass grafting with expanded polytetrafluoroethylene: 12-year patency. Ann. Thorac. Surg. 65:1442-1444, 1998.

${ }^{34}$ Meyers, S. R., P. T. Hamilton, E. B. Walsh, D. J. Kenan, and M. W. Grinstaff. Endothelialization of titanium surfaces. Adv. Mater. 19:2492-2498, 2007.

${ }^{35}$ Meyers, S. R., D. J. Kenan, and M. W. Grinstaff. Enzymatic release of surface adsorbed rgd therapeutic from a cleavable peptide anchor. ChemMedChem 3:1645-1648, 2008.

${ }^{36}$ Meyers, S. R., K. Xiaojuan, X. Huang, E. B. Walsh, M. W. Grinstaff, and D. J. Kenan. The development of peptidebased interfacial biomaterials for generating biological functionality on the surface of bioinert materials. Biomaterials 30:277-285, 2009.

${ }^{37}$ Niklason, L. E., J. Gao, W. M. Abbott, K. Hirschi, S. Houser, R. Marini, and R. Langer. Functional arteries grown in vitro. Science 284:489-493, 1999.

${ }^{38}$ Olivieri, M. P., and K. S. Tweden. Human serum albumin and fibrinogen interactions with an adsorbed rgd-containing peptide. J. Biomed. Mater. Res. 46:355-359, 1999.

${ }^{39}$ Pierschbacher, M. D., and E. Ruoslahti. Cell attachment activity of fibronectin can be duplicated by small synthetic fragments of the molecule. Nature 309:30-33, 1984.
${ }^{40}$ Plow, E. F., T. A. Haas, L. Zhang, J. Loftus, and J. W. Smith. Ligand binding to integrins. J. Biol. Chem. 275:21785-21788, 2000.

${ }^{41}$ Quirk, R. A., W. C. Chan, M. C. Davies, S. J. Tendler, and K. M. Shakesheff. Poly(l-lysine)-grgds as a biomimetic surface modifier for poly(lactic acid). Biomaterials 22: 865-872, 2001.

${ }^{42}$ Ratner, B. D., and S. J. Bryant. Biomaterials: where we have been and where we are going. Ann. Rev. Biomed. Eng. 6:41-75, 2004

${ }^{43}$ Ratner, B. D., A. S. Hoffman, F. J. Schoen, and J. E. Lemons. Biomaterials Science: An Introduction to Materials in Medicine. San Diego: Academic Press, 2000.

${ }^{44}$ Reed, A. M., and D. K. Gilding. Biodegradable polymers for use in surgery-poly(glycolic)-poly(lactic acid) homo and co-polymers. 2. In vitro degradation. Polymer 22: 494-498, 1981.

${ }^{45}$ Ruoslahti, E. Rgd and other recognition sequences for integrins. Annu. Rev. Cell. Dev. Biol. 12:697-715, 1996.

${ }^{46}$ Sanghvi, A. B., K. P. Miller, A. M. Belcher, and C. E. Schmidt. Fabricating novel biomimetic polymers using combinatorial peptide screening technologies. Abstracts of Papers of the American Chemical Society 227:U126, 2004.

${ }^{47}$ Sanghvi, A. B., K. P. Miller, A. M. Belcher, and C. E. Schmidt. Biomaterials functionalization using a novel peptide that selectively binds to a conducting polymer. Nat . Mater. 4:496-502, 2005.

${ }^{48}$ Shin, H., S. Jo, and A. G. Mikos. Biomimetic materials for tissue engineering. Biomaterials 24:4353-4364, 2003.

${ }^{49}$ Smith, G. Filamentous fusion phage: novel expression vectors that display cloned antigens on the virion surface. Science 228:1315-1317, 1985.

${ }^{50}$ Van Der Flier, A., and A. Sonnenberg. Function and interactions of integrins. Cell Tissue Res. 305:285-298, 2001.

${ }^{51}$ Varani, J., D. R. Inman, S. E. Fligiel, and W. J. Hillegas. Use of recombinant and synthetic peptides as attachment factors for cells on microcarriers. Cytotechnology 13:89-98, 1993.

${ }^{52}$ Vroman, L. Effect of absorbed proteins on the wettability of hydrophilic and hydrophobic solids. Nature 196: 476-477, 1962.

${ }^{53}$ Wallace, C. S., J. C. Champion, and G. A. Truskey. Adhesion and function of human endothelial cells co-cultured on smooth muscle cells. Ann. Biomed. Eng. 35:375-386, 2007.

${ }^{54}$ Walsh, E. B., C. Middleton, M. J. Davis, D. J. Kenan, and M. W. Grinstaff. Multifunctional peptides as interfacial biomaterials. ACS Div. Polym. Chem. 43:753, 2002.

${ }^{55}$ Williams, D. F. On the mechanisms of biocompatibility. Biomaterials 29:2941-2953, 2008. 
Reproduced with permission of the copyright owner. Further reproduction prohibited without permission. 